\title{
Prolonged electrical stimulation-induced gluteal and hamstring muscle activation and sitting pressure in spinal cord injury: Effect of duty cycle
}

\author{
Christof A. J. Smit, MD; ${ }^{*}$ Karin J. A. Legemate, MSc; ${ }^{2}$ Anja de Koning, MSc; ${ }^{2}$ Sonja de Groot, PhD; ${ }^{1,3}$ \\ Janneke M. Stolwijk-Swuste, MD, PhD; ${ }^{1}$ Thomas W. J. Janssen, PhD $^{1-2}$ \\ ${ }^{1}$ Amsterdam Rehabilitation Research Centre, Reade, Amsterdam, the Netherlands; ${ }^{2}$ Research Institute MOVE, Faculty \\ of Human Movement Sciences, VU University, Amsterdam, the Netherlands; ${ }^{3}$ Centre for Human Movement Sciences, \\ University Medical Centre, University of Groningen, Groningen, the Netherlands
}

\begin{abstract}
Pressure ulcers (PUs) are highly prevalent in people with spinal cord injury (SCI). Electrical stimulation (ES) activates muscles and might reduce risk factors. Our objectives were to study and compare the effects of two duty cycles during $3 \mathrm{~h}$ of ES-induced gluteal and hamstring activation on interface pressure distribution in sitting individuals with SCI and study the usability of a newly developed electrode garment (ES shorts). Ten individuals with SCI participated in this study, in which two ES protocols with different duty cycles $(1: 1 \mathrm{~s}$ vs $1: 4 \mathrm{~s}$ on-off) were applied in counterbalanced order using a custom-made garment with built-in electrodes. Outcome variables included interface pressure of the ischial tuberosities (ITs) and pressure gradient. A questionnaire was used to determine usability of the ES shorts. In both protocols, ES caused a significant decrease in average IT pressure compared with rest (no ES); on average, 35\% for protocol 1:4 and 13\% for protocol 1:1. The ES on-off duty cycle of protocol 1:4 showed less muscle fatigue. In general, participants scored the usability of the ES shorts as satisfactory. In this study, the application of ES resulted in a significant decrease in IT pressure. The ES on-off duty cycle of $1: 4 \mathrm{~s}$ is recommended because of the less fatiguing effect. ES of the hamstrings and gluteal muscles might be a promising method in preventing PUs, but further study is needed.
\end{abstract}

Key words: duty cycle, electrical stimulation, interface pressure, ischial tuberosity, muscles, pressure sores, pressure ulcers, questionnaire, sitting pressure, spinal cord injury.

\section{INTRODUCTION}

Pressure ulcers (PUs) are the most prevalent secondary complications in individuals with spinal cord injury (SCI) [1-2]. Throughout their lives, up to 80 percent of people with SCI experience a PU [1-2], leading to radical consequences such as decreased mobility and independence, delayed rehabilitation, and exclusion from social activities [2-3]. This has a tremendous effect on the individual's physical and psychological condition [3-4]. The consequences also result in high costs for the community [4-5].

Prevention has focused on cushions to improve interface pressure distribution and pressure-relief movements [2-3]. Although interface pressure can be reduced with these methods, they are inadequate in completely preventing PUs [2-3]. Moreover, these are merely passive methods that do not activate the muscles, which therefore do not ameliorate intrinsic risk factors for developing PUs such as muscle atrophy and decreased circulation.

\footnotetext{
Abbreviations: ANOVA $=$ analysis of variance, ASIA $=$ American Spinal Injury Association, ES = electrical stimulation, $\mathrm{FSA}=$ force sensitive array, $\mathrm{IT}=$ ischial tuberosity, $\mathrm{PU}=$ pressure ulcer, $\mathrm{SCI}=$ spinal cord injury.

*Address all correspondence to Christof A. J. Smit, MD; Reade, Spinal Cord Unit, Location Overtoom, PO Box 58271, 1040 HG Amsterdam, the Netherlands; 003-120607-1679; fax: 003-120-607-1891. Email: c.smit@reade.nl http://dx.doi.org/10.1682/JRRD.2012.07.0134
} 
Another potentially useful method for prevention of PUs is muscle activation by electrical stimulation (ES), which has been found to improve both interface pressure distribution and intrinsic risk factors, indicated by Levine et al. [6-7], Levine and Kett [8], and others [3-5,9-10]. In previous studies, we demonstrated that surface ES of the gluteal muscles temporarily decreases interface pressure below ischial tuberosities (ITs) [11-12]. Activation causes a change in muscle shape and tone and leads the pressure away from the ITs in the direction of the knees. However, van Londen et al. [12] included only a $30 \mathrm{~min}$ ES protocol with self-adhesive electrodes under the buttocks using a stimulator not suitable for daily life. Furthermore, no effects were studied for a longer period or using different protocols. It was unclear whether muscle fatigue would occur after a longer period of ES and whether this would influence the pressure-relieving effects of ES. In a subsequent study, we found that a $1 \mathrm{~h}$ activation protocol of both hamstrings and gluteal muscles gives more pressure relief from the ITs than activation of the gluteal muscles only [12]. In this study, we discuss the desirable prolonged activation of these muscles. ES for a longer period outside the laboratory would be more similar to daily life and possibly generate muscle fatigue. In an interesting single-case study using implanted electrodes, Bogie et al. [3] described an on-off duty cycle of ES of $1: 1 \mathrm{~s}$ in a $3 \mathrm{~min}$ period, with a $17 \mathrm{~min}$ interstimulation interval, approximating the frequency of weight shifting recommended for wheelchair users at risk of tissue breakdown $[3,7,13]$. This dynamic stimulation regime could be used for up to $10 \mathrm{~h}$ a day while sitting in the wheelchair. However, this duty cycle has not been compared with other protocols; therefore, it is not clear whether this is the optimal stimulation-rest ratio.

In the present study, we intended to study the effects of a longer stimulation period $(3 \mathrm{~h})$ than in previous studies, where acute effects, $30 \mathrm{~min}$ or $1 \mathrm{~h}$, of ES have been studied [11-12]. We compared the same stimulation-rest interval $(1: 1 \mathrm{~s})$ during the $3 \mathrm{~min}$ of ES with a different protocol $(1: 4 \mathrm{~s})$. With this interval, activated muscles would be allowed more time to recover from contractions. Dreibati et al. showed that increasing the rest time improves the force of muscle contractions and decreases fatigue, which is of course beneficial [14]. We hypothesized that, as muscle contractions will be more effective over a longer period of time when muscle fatigue does not occur, more rest might have more positive effects on pressure relief. In the present study, we used previously used, specially developed ES shorts with built-in elec- trodes connected to a portable stimulator. They have only been used in a previous study [11] and not in clinical practice. We hypothesized that, in general, the usability would be satisfactory.

Therefore, the aims of this study were to determine-

1. The effect of $3 \mathrm{~h}$ of ES-induced gluteal and hamstring activation on interface pressure distribution in sitting individuals with SCI (vs 30 [11] and $60 \mathrm{~min}$ [12]).

2 . The effects of duty cycle (1:1 s vs $1: 4 \mathrm{~s})$ on interface pressure and subsequent muscle fatigue over time.

3. The usability of the electrostimulation garment (ES shorts).

\section{MATERIALS AND METHODS}

\section{Participants}

Ten individuals with SCI having a complete or incomplete upper motor neuron lesion (American Spinal Injury Association [ASIA] A, B, or C) aged between 18 and $70 \mathrm{yr}$ were recruited for this study, approved by the local medical ethics committee. Gluteal and hamstring muscles, skin under the buttocks, and spinal reflexes had to be intact, which one physician evaluated for all participants. Previous (plastic) surgery under the buttock area was not a contraindication for inclusion. Exclusion criteria included individuals with a flaccid paralysis and areflexia, a history of severe autonomic dysreflexia, current PUs under the ITs or in the sacral region, severe cognitive or communicative disorders, intolerance for ES, or any other contraindication for ES. The participants provided written informed consent. Table 1 shows participant characteristics.

\section{Study Design}

The two $3 \mathrm{~h}$ protocols with different duty cycles were performed on two separate days in a counterbalanced order. Every participant had to put on the ES shorts (either individually or with assistance) and could wear normal pants over the ES shorts. Figure 1 shows the ES shorts with electrical stimulator. Each participant used his or her own wheelchair with daily use cushion. The ES protocol lasted $3 \mathrm{~h}$, with interface pressure measurement sessions three times per hour, during the last minute of both rest and stimulation periods. Between the measurement sessions, participants were allowed to perform normal daily activities in and around the rehabilitation center. Finally, on the second (and last) day of the ES protocol, all participants completed a questionnaire on the usability of the ES shorts consisting of seven questions (Table 2). 
Table 1.

Participant characteristics $(n=10)$.

\begin{tabular}{lc}
\hline \multicolumn{1}{c}{ Characteristic } & n or Mean \pm SD (range) \\
\hline Sex & 7 \\
Male & 3 \\
Female & $40.6 \pm 12.8(26-58)$ \\
Age (yr) & 7 \\
Lesion Level & 3 \\
C3-C8 & 6 \\
Th1-Th12 & 3 \\
Impairment Classification & 1 \\
ASIA A & $162 \pm 99(85-309)$ \\
ASIA B & $83.2 \pm 15.4(70-107)$ \\
ASIA C & \\
Time Since Injury (mo) & 3 \\
Body Mass (kg) &
\end{tabular}

\section{Electrical Stimulation with ES Shorts}

A stimulator (NeuroPro 8 channel, Axiobionics; Ann Arbor, Michigan) connected to the custom-made ES shorts (Axiobionics) was used to apply ES. These ES shorts are made of partially elastic Lycra material containing flat embedded surface electrodes with built-in leads connected to the portable stimulator to noninvasively activate the gluteal muscles and hamstrings. Although participants' (a)

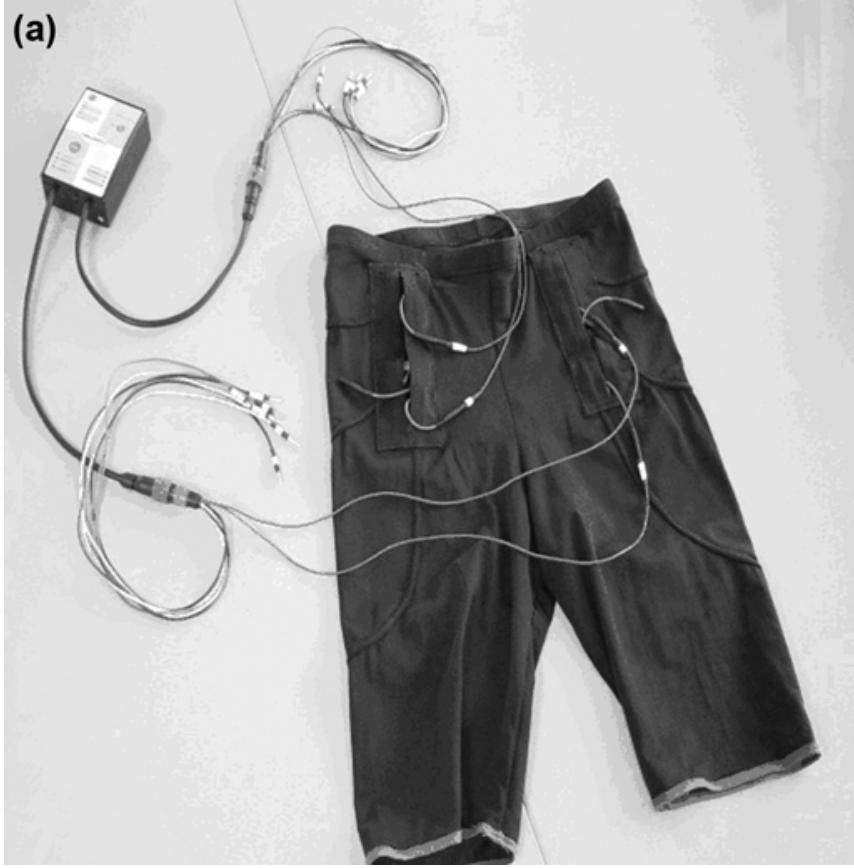

(b)

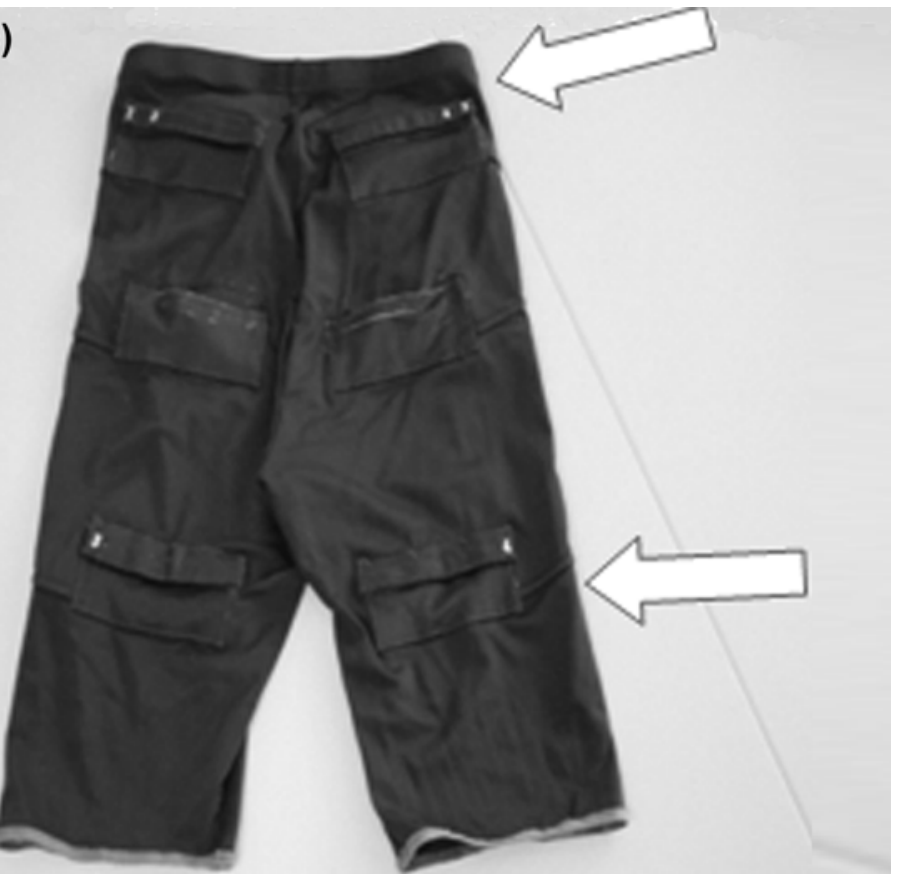

Figure 1.

Electrical stimulation shorts. (a) Front side with embedded electrodes and NeuroPro electrical stimulator. (b) Back side, where two arrows indicate electrodes used. 
Table 2.

Questionnaire on usability of electrical stimulation shorts.

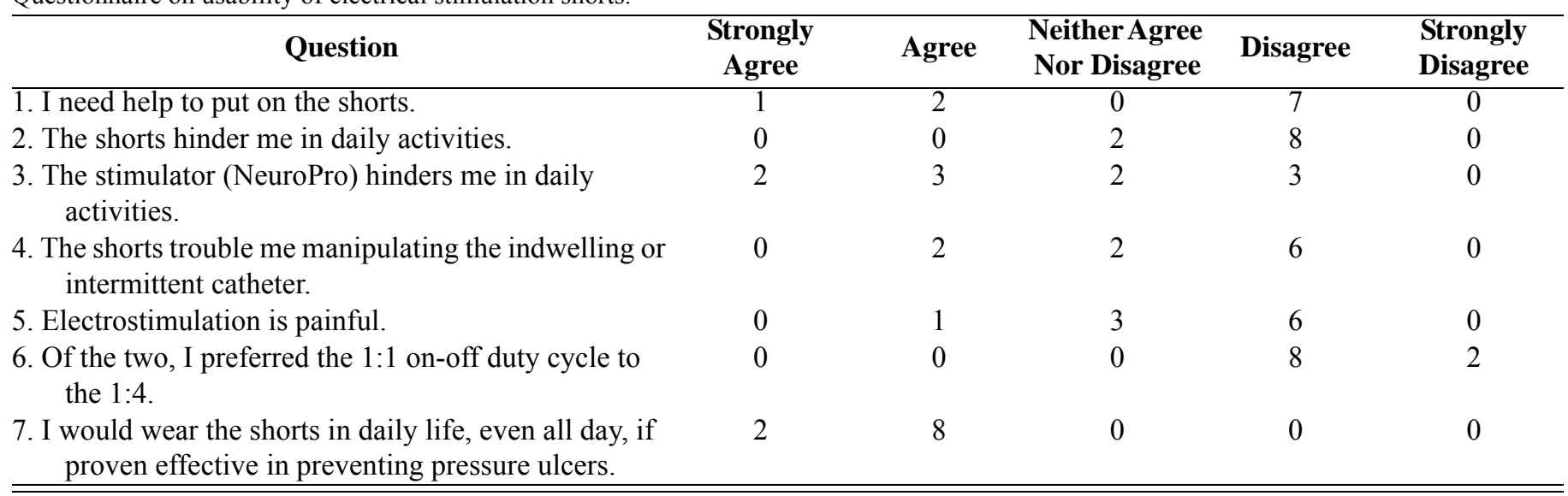

body mass ranged from 70 to $107 \mathrm{~kg}$ (Table 1), the ES shorts fit them all. Electrodes align automatically over the gluteal and hamstring muscles as the ES shorts are put on, with one electrode positioned at the upper (proximal) part of the gluteal muscle above the sitting area and one about halfway down the hamstrings area, preventing the participant from sitting on the electrodes or wires while wearing the shorts. The portable stimulator was attached around the subject's waist with a strap. Ultrasound gel was applied over the electrodes and ES was delivered at a standard constant voltage of $150 \mathrm{~V}$. Using $50 \mathrm{~Hz}$ biphasic impulse frequency, a tetanic contraction was induced. The current amplitude resulting in the best pressure reduction was determined for each participant by increasing the amplitude in steps of 5 to $10 \mathrm{~mA}$ to a maximum, without discomfort or excessive muscle contractions that would disturb normal sitting. This amplitude was subsequently used for this participant for both protocols. Protocol 1:1 had a duty cycle of $1 \mathrm{~s}$ ES and $1 \mathrm{~s}$ rest, while protocol 1:4 had an ES-rest ratio of $1 \mathrm{~s}$ on and $4 \mathrm{~s}$ off. The ES was applied for $3 \mathrm{~min}$, followed by a $17 \mathrm{~min}$ rest period. This was repeated until $3 \mathrm{~h}$ of stimulation and rest were completed.

\section{Interface Pressure Measurements}

Interface pressure distribution was measured using a pressure-mapping device (force sensitive array [FSA], Vista Medical; Winnipeg, Canada), a $2 \mathrm{~mm}$ thick soft flex mat of $42 \times 42 \mathrm{~cm}$ consisting of 256 pressure sensors, placed between the cushion on the wheelchair and the buttocks of the participant. Before testing, it was calibrated between 0 and $200 \mathrm{~mm} \mathrm{Hg}$ according to the system's calibration protocol.
Each participant sat in a "normal position" with feet on the wheelchair footrests, arms on the armrests (if any) or on his or her lap, and the lower back against the backrest. The individual sat in his or her wheelchair on the FSA for 5 min to allow the cushion to adapt to the participant's buttocks. Subsequently, the protocol was started and outcome variables were obtained during ES and in the last minute of rest (just prior to ES). The two IT areas were defined as the $3 \times 3$ sensors with the highest pressure values. The values of the nine sensors were averaged to result in the IT pressure for left and right IT area. The pressure gradient was calculated by subtracting the mean pressure from the (average) values of the 9 sensors in the tuber area and the 16 surrounding sensor values (Figure 2). The pressure gradient may indicate shear forces $[2,12]$. Higher shear forces cause a higher risk of tissue damage and PUs $[1-2,6,15]$.

Pressure values were recorded during the $3 \mathrm{~min}$ of ES and during the last minute of the preceding rest. This produced four FSA recordings per hour, namely T0 (before start), T1, T2, and T3. Three pressure recordings within each time point and one mean value were reported for each time point. Figure 3 shows measurements within $1 \mathrm{~h}$.

\section{Data Analysis}

IT pressure and pressure gradient, collected with the FSA, were calculated by averaging the values for right and left buttock. The acute effects of ES were investigated by calculating delta $(\Delta)$ IT pressure ( $\Delta$ IT Pressure), subtracting the lowest pressure value of all stimulation moments during $3 \mathrm{~min}$ of ES from the average pressure values at rest at the different moments in time (i.e., T0, T1, T2, or T3). Delta $(\Delta)$ pressure gradient ( $\Delta$ Gradient) 
(a)

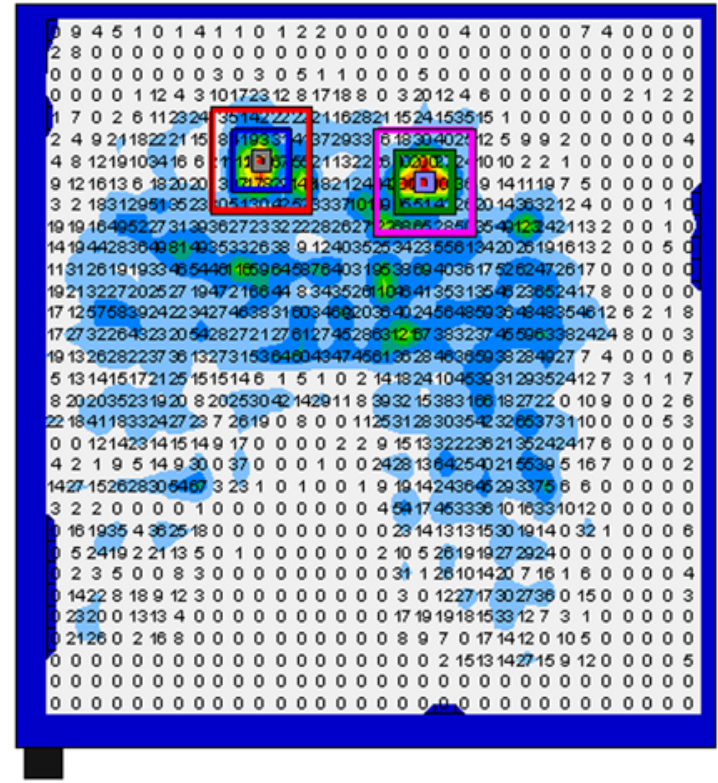

(b)

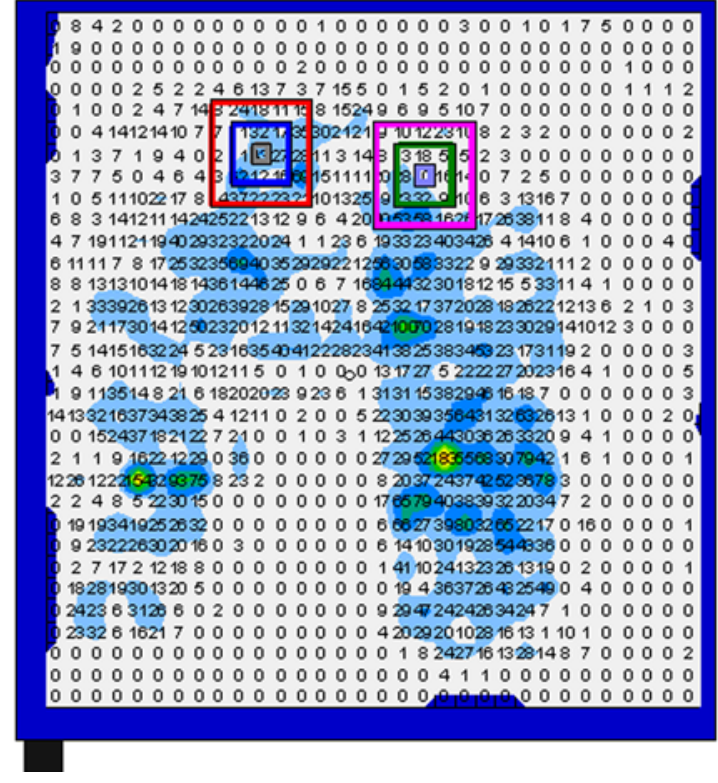

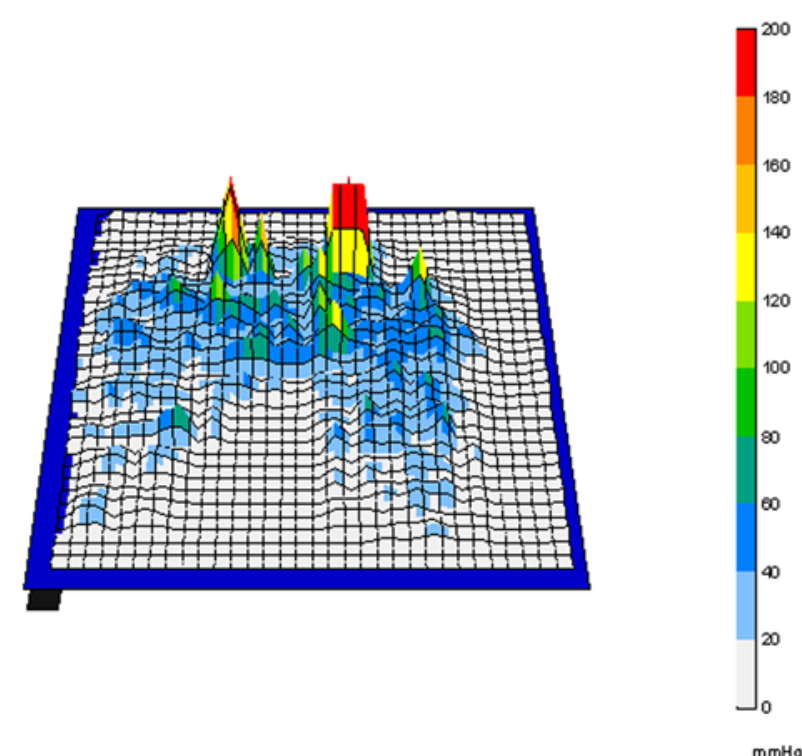

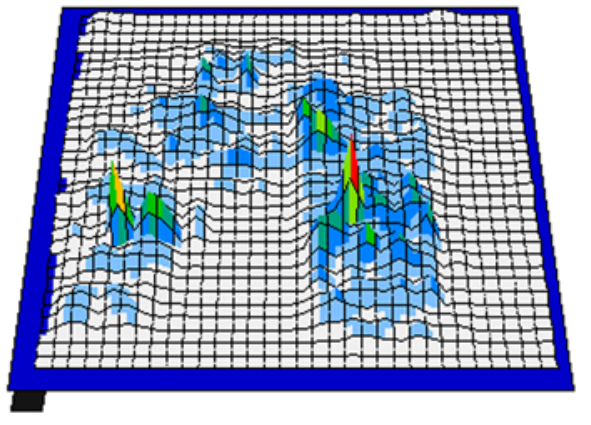

$\mathrm{mmHg}$

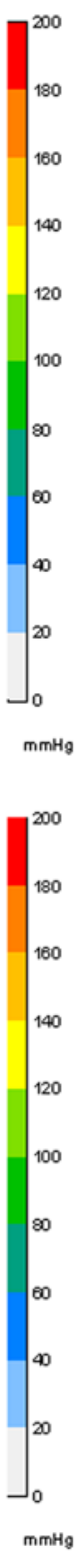

$\mathrm{mmHg}$

Figure 2.

Force sensitive array frame of sitting pressure distribution during (a) rest and (b) with electrical stimulation of gluteal and hamstring muscles for individual participant. Squares are used to determine pressure of ischial tuberosities and pressure gradient.

was calculated subtracting the mean values of the pressure gradient with ES from average pressure values at rest. This resulted in variables $\Delta \mathrm{IT}$ Pressure and $\Delta$ Gradient at T0, T1, T2, and T3 for both protocols.

When fatigue occurs, contractions will be less forceful, which is expected to result in a diminished $\Delta$ IT Pressure and $\Delta$ Gradient [14]. To establish whether muscle fatigue had occurred, interface pressure during the last three stimulations of the 3 min ES period at T3 was compared with pressure values in rest at T0. Differences were also calculated at T0, T1, T2, and T3 for both protocols.

\section{Statistical Analysis}

SPSS version 16.0 (IBM Corporation; Armonk, New York) was used to analyze the data. All results were described as mean \pm standard deviation. Repeated measures 


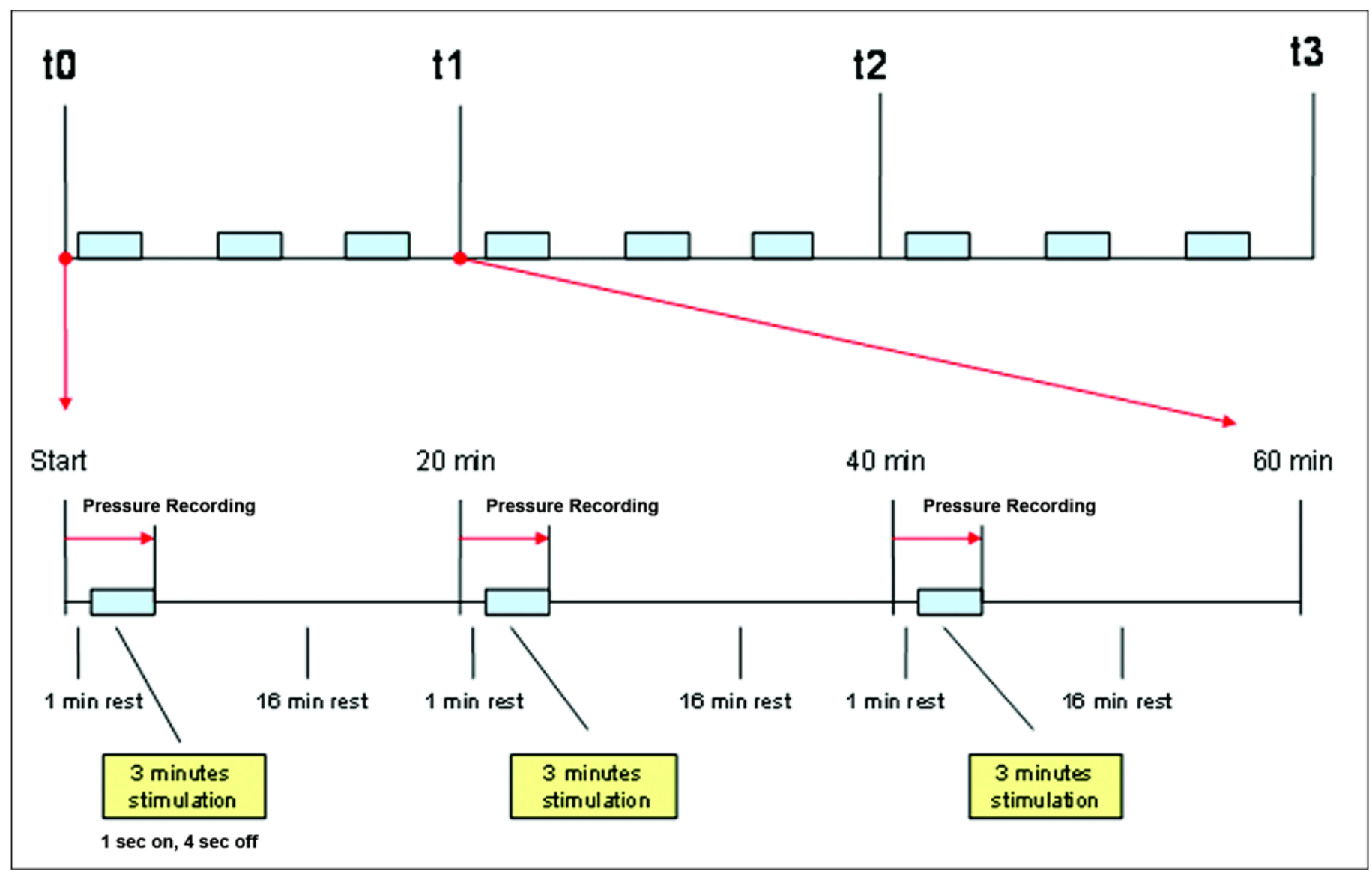

Figure 3.

Time (t) table stimulation protocol (1:4 s). Blue blocks indicate electrical stimulation is "on." Red arrows indicate pressure mapping.

analysis of variance (ANOVA) was used to analyze data (IT pressure and pressure gradient) within the protocols (1:1 and 1:4 s). A $2 \times 4$ within-protocol general linear model ANOVA with repeated measures $(2$ protocols $\times$ 4 moments in time) was used to compare data of the two protocols over time. Differences with a $p$-value of $\leq 0.05$ were considered significant. Table 2 presents the answers to the questionnaire.

\section{RESULTS}

\section{Effects of 3 Hours of Electrical Stimulation on Interface Pressure}

All participants were stimulated with ES ranging from $70 \mathrm{~mA}$ to a maximum of $115 \mathrm{~mA}$. Both protocols resulted in an acute significant decrease of pressure during ES compared with rest (no ES) for all measured val- ues, i.e., IT pressure and pressure gradient (Table 3). For example, IT pressure decreased at T0 from 106.0 to $37.2 \mathrm{~mm} \mathrm{Hg}$ (39\%) (protocol 1:1) and from 103.0 to $31.2 \mathrm{~mm} \mathrm{Hg} \mathrm{(32 \% )} \mathrm{(protocol} \mathrm{1:4).} \mathrm{Muscles} \mathrm{fatigued} \mathrm{only}$ after protocol $1: 1$ as $\Delta \mathrm{IT}$ Pressure tended to decrease over time (within-protocol, $p=0.06$ ).

\section{Differences Between Protocols}

IT pressure and pressure gradient during ES compared with rest were not significantly different between protocols (time within protocol), although there was a tendency for larger reductions with protocol 1:4 than for protocol 1:1. Over time, protocol 1:4 had significantly more effect than protocol 1:1 for $\Delta \mathrm{IT}$ Pressure $(p=0.04$, Table 3). While effects reduced in protocol 1:1 (i.e., less pressure reduction), the effects remained stable in protocol 1:4 (Figure 4). This was not the case for $\Delta$ Gradient $(p=0.33)($ time $\times$ protocol). Fatigue occurred after the 
Table 3.

Differences $(\Delta$, mean \pm standard deviation) in pressure of ischial tuberosities (ITs) and pressure gradient between electrical stimulation (ES)induced activation and at rest and end of blocks T0, T1, T2, and T3. Also, differences in pressure in very last 3 min of ES-induced activation at T3 compared with rest at $\mathrm{T} 0$ for both protocols (1:1 and 1:4 s).

\begin{tabular}{|c|c|c|c|c|c|c|c|}
\hline \multirow{2}{*}{ Pressure } & \multirow{2}{*}{ Protocol } & \multicolumn{4}{|c|}{ Time } & \multirow{2}{*}{$\begin{array}{c}\text { Time } \\
\text { (within protocol) } \\
p \text {-Value }\end{array}$} & \multirow{2}{*}{$\begin{array}{c}\text { Time } \times \text { Protocol } \\
p \text {-Value }\end{array}$} \\
\hline & & T0 & $\mathbf{T 1}$ & T2 & T3 & & \\
\hline \multirow{2}{*}{$\begin{array}{l}\Delta \mathrm{IT} \text { Pressure } \\
\quad(\mathrm{mm} \mathrm{Hg})\end{array}$} & $1: 1$ & $-37.2 \pm 44.2$ & $-12.7 \pm 13.1$ & $-16.1 \pm 14.6$ & $-9.0 \pm 19.2$ & 0.06 & $0.04^{*}$ \\
\hline & $1: 4$ & $-31.2 \pm 44.2$ & $-33.2 \pm 19.8$ & $-29.5 \pm 18.2$ & $-36.4 \pm 20.5$ & 0.62 & - \\
\hline \multirow{2}{*}{$\begin{array}{l}\Delta \text { Gradient } \\
\quad(\mathrm{mm} \mathrm{Hg})\end{array}$} & $1: 1$ & $-18.8 \pm 33.9$ & $-7.1 \pm 9.3$ & $-8.2 \pm 10.0$ & $-5.7 \pm 11.2$ & 0.06 & $0.04^{*}$ \\
\hline & $1: 4$ & $-16.1 \pm 11.2$ & $-22.5 \pm 19.7$ & $-14.2 \pm 18.8$ & $-18.3 \pm 17.9$ & 0.62 & - \\
\hline \multirow{2}{*}{$\begin{array}{l}\Delta \mathrm{IT} \text { Pressure } \\
\quad 3 \mathrm{~min}(\mathrm{~mm} \mathrm{Hg})\end{array}$} & $1: 1$ & $-18.1 \pm 16.5$ & $-5.4 \pm 10.7$ & $-8.3 \pm 9.9$ & $-2.4 \pm 13.9$ & 0.06 & $0.04^{*}$ \\
\hline & $1: 4$ & $-10.3 \pm 5.4$ & $-12.1 \pm 6.8$ & $-10.2 \pm 11.0$ & $-14.7 \pm 12.5$ & 0.62 & - \\
\hline \multirow{2}{*}{$\begin{array}{l}\Delta \text { Gradient } \\
\quad 3 \min (\mathrm{mm} \mathrm{Hg})\end{array}$} & $1: 1$ & $-4.1 \pm 8.8$ & $-3.0 \pm 6.1$ & $-4.0 \pm 5.6$ & $-2.3 \pm 7.1$ & 0.06 & $0.04^{*}$ \\
\hline & $1: 4$ & $-3.1 \pm 7.1$ & $-5.8 \pm 20.5$ & $-3.3 \pm 11.5$ & $-5.1 \pm 10.2$ & 0.62 & - \\
\hline
\end{tabular}

application of protocol 1:1 more than protocol 1:4, because in the last $3 \mathrm{~min}$ of ES, there is a significant difference between protocols for pressure reduction $(p<$ $0.001)$ over time.

\section{Usability of ES Shorts}

All participants completed the questionnaire after the measurements (Table 2). Of the 10 participants, 3 were not able to put on the ES shorts without help; their lesion was at cervical level 6 or higher. None of the participants found the ES shorts to interfere with daily activities (such as riding the wheelchair), but the stimulator did hinder five of the participants in daily activities. Six participants had an indwelling catheter and two of them perceived the ES shorts to hinder the working of the catheter while another two indicated that the ES shorts were not comfortable in combination with their catheter. Only one participant mentioned the ES to be painful. Nobody had trouble sustaining the 3 min ES periods. All participants reported that they experienced protocol 1:4 as the more comfortable of the two. Finally, if the ES shorts were found helpful in preventing PUs, all of the participants stated they would be willing to use them all day in daily life.

\section{DISCUSSION}

The purpose of this study was to investigate the effect of two protocols of surface ES on the interface pressure distribution in sitting individuals with SCI tested over a (prolonged) $3 \mathrm{~h}$ period. This is a longer period than in previous studies and more comparable with a daily life situation $[12,16]$. We hypothesized that because muscle contractions will be more effective over a longer period of time when muscle fatigue does not occur, more rest might have more positive effects on pressure relief. The results in this study showed clear reductions in IT pressure and pressure gradient of the buttocks during ES, which is in line with previous studies [2-3,10,12,16-18]. During ES, an increase in pressure was seen in the area of the upper legs as the pressure was relocated from the ITs to the front of the sitting surface. This (desirable) relocation did not create any skin problems because there are no bony prominences at the hamstring site near the knees.

Bogie et al. [3] described an on-off ES duty cycle of $1: 1 \mathrm{~s}$ in a 3 min period, with a $17 \mathrm{~min}$ interstimulation interval, approximating the frequency of weight shifting recommended for wheelchair users at risk of tissue breakdown $[3,8,10]$. This duty cycle has not been compared with other protocols; therefore, it is not clear whether this is the optimal ES-rest ratio. We have compared the same ES-rest interval (protocol 1:1) during the 3 min of ES with a different protocol (protocol 1:4). Comparison of these two ES on-off duty cycle protocols revealed that protocol 1:4 had preferable effects over protocol 1:1. We showed that over $3 \mathrm{~h}$ of stimulation in this protocol (1:4 s), participants' gluteal and hamstring muscles kept contracting without marked muscle fatigue. This suggests that perfusion, as well as oxygenation, was sufficient during the $3 \mathrm{~h}$ of testing. This is also found in 
JRRD, Volume 50, Number 7, 2013

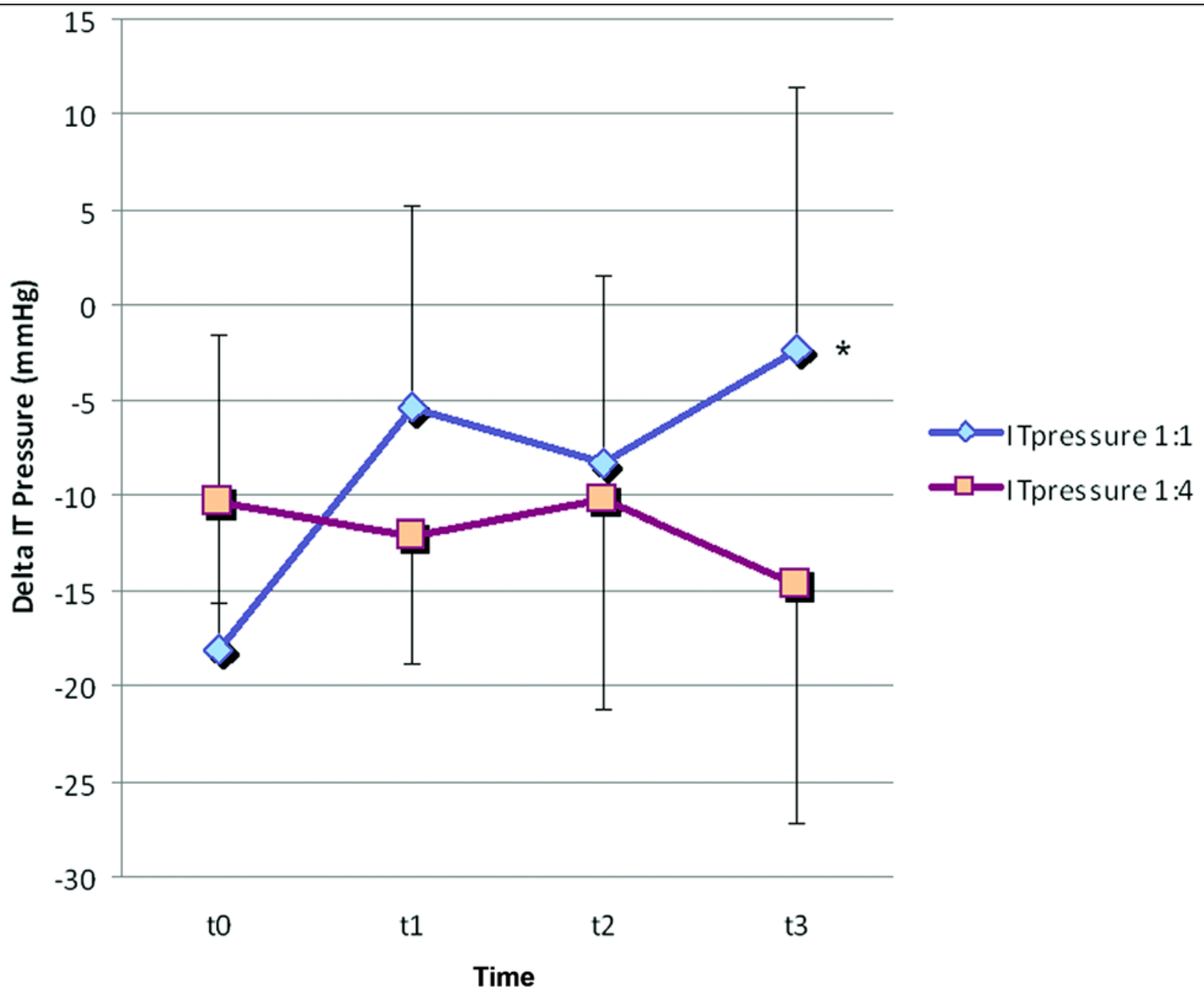

Figure 4.

Delta pressure of ischial tuberosities (ITs) (difference between IT pressure in rest and IT pressure with electrical stimulation [ES]) for both protocols at t (time) 3, last 3 min of ES. Significant difference exists between protocols (1:1 and 1:4 s) at t3, probably because of muscle fatigue after applying protocol 1:1.

Gerrits et al. [9], Janssen et al. [15], and Janssen and Hopman [19], showing the improvement of blood flow and oxygenation in stimulated paralyzed muscles of people with SCI.

The decline of the pressure relief effect over time in protocol 1:1 is likely caused by less forceful contractions due to muscle fatigue after repetitive activation [14]. We hypothesize that ES-induced training of these muscles will have a positive effect on fatigue. Muscles in good condition will be better perfused and oxygenated and will likely continue to contract after prolonged activation. We do not know whether another duty cycle (for example, on-off ratio of 1:6 s) and/or rest period (for example, shorter than $17 \mathrm{~min}$ ) will have even more preferable effects on pressure relief versus fatigue [14]. How much recovery time do muscles need for continuous activation after ES? In the literature, no studies were found describing effects of ES training on fatigue or sitting pressure in people with SCI or other diagnosed groups. This needs further investigation. In addition, after comparing the effects of both protocols, all participants reported that they experienced protocol 1:4 as more comfortable in the usability questionnaire. In conclusion, protocol 1:4 overall objectively and subjectively had preferable effects over protocol 1:1. 
Comparing our results with other studies like Bogie et al. $[3,20]$ shows that our results are comparable for the conclusion that ES has pressure-reducing effect on the ITs. Bogie et al. used a different implanted ES system to activate the gluteal muscles [3]. van Londen et al. also used transcutaneous ES, but activated gluteal muscles only [12], as opposed to this study, where gluteal and hamstring muscles were activated. The pressure gradient reduced $14.0 \mathrm{~mm} \mathrm{Hg}$ in van Londen et al., while in the present study, the pressure gradient reduced $9.0 \mathrm{~mm} \mathrm{Hg}$. This difference may be caused by the use of a different ES protocol, because van Londen et al. stimulated with an ES-rest cycle of $0.5 \mathrm{~s}$ on and $15 \mathrm{~s}$ off, and also by individually positioned electrodes for each participant; in the present study, we used the specially developed ES shorts with built-in electrodes connected to a portable stimulator. It has only been used in our previous study [11], not in clinical practice. This method is noninvasive, the costs are relatively low, and electrodes are fabricated within the ES shorts and therefore do not have to be placed repeatedly. The stimulator we used is relatively small and easily portable but hindered 5 of the 10 participants. A challenge for the future is to improve this stimulator, most importantly by reducing the size and weight. Also, this new method has a major advantage over systems such as dynamic seating systems $[10,18]$ in that it can be used independently from the wheelchair. The ES system "travels" with the person without the need to make any changes or arrangements. It can be used while sitting on the couch, on a plane during a $10 \mathrm{~h}$ flight, while sitting in a sports wheelchair, etc. In addition to that advantage, no lifting is needed, which reduces the load on the shoulders and the risk of shoulder and arm injuries [11]. Moreover, the ES shorts are worn under regular clothes [21]. Although one participant (ASIA B; sensible incomplete) reported that ES was painful and some participants reported practical problems, such as applying the ultrasound gel or handling the stimulator, none reported other relevant side effects while receiving ES with the ES shorts. We hypothesized that the usability of the ES shorts in general would be satisfactory, which has been the case. The ES shorts were found to be feasible for the application of ES in daily life situations. Participants all stated they would wear the ES shorts with the stimulator in daily life, even all day long, if they helped prevent PUs.

It will be necessary to determine which reduction of pressure is clinically relevant and therefore how often the ES shorts should be worn to prevent PUs. Circulation and oxygenation measurements in the gluteal and hamstring area are also interesting to deter effects of surface ES on tissue deeper under the stimulated surface. Future research has also revealed the possible additional effects of surface ES on reduction of muscle atrophy. Levine et al. $[6-7,17]$ and Levine and Kett [8] already showed that tissue shape changed and muscle hypertrophy occurred after muscle ES.

There were two study limitations. One is the FSA map used to record sitting pressure. This pressure map measures surface pressure. We know that PUs occur in deep tissue. In the literature, no studies have been found describing the relation between surface (sitting) pressure and deep-tissue cell deformation. The other study limitation is the ultrasound gel used in the ES shorts to conduct ES pulses through the skin. One potential participant resigned after being shown how to use the ultrasound gel in the ES shorts. The gel caused "wet" spots in his clothes, which he found undesirable.

Unfortunately, the method of activating muscles used in this study is not suitable for persons with a flaccid paresis such as in cauda equine syndrome. Also, intact sensibility might sometimes be a problem; however, in our study, the two participants who had incomplete lesions with partially intact sensibility did not perceive the ES as painful or even unpleasant. One participant did find the stimulation unpleasant. She had a complete (ASIA A) lesion but with a zone of partial (preserved) innervation, causing painful sensations above a certain stimulation level.

For further research, we recommend customized ES shorts for each individual participant. This is important because compliance wearing the shorts is of vital importance. As for the clinical implications, applying ES seems a promising method but the feasibility and usability of the ES shorts need to be optimized. In a controlled setting such as Department of Veterans Affairs hospital or rehabilitation clinic, conventional stimulators with electrodes could also be used in the future to apply ES instead of using the ES shorts although we believe the ES shorts provide more advantages than this method. Future research has to reveal long-term effects of ES on circulation and oxygenation and which pressure reduction actually decreases the incidence of PUs. The usability of the ES shorts can also be improved, especially with regard to the ultrasound gel. Results so far are promising and suggest that surface ES might help prevent PUs in sitting individuals with SCI. 


\section{CONCLUSIONS}

ES applied to the gluteal and hamstring muscles in a custom-made electrode garment with built-in electrodes gives significant pressure relief to ITs even after $3 \mathrm{~h}$ of activation in individuals with SCI. An on-off ES ratio of $1: 4 \mathrm{~s}$ versus $1: 1 \mathrm{~s}$ gave better results in pressure relief during a $3 \mathrm{~h}$ protocol, without marked muscle fatigue. In general, participants scored the usability of the ES shorts as satisfactory, and all stated they would wear them daily should they help prevent PUs.

\section{ACKNOWLEDGMENTS}

\section{Author Contributions:}

Study concept and design: C. A. J. Smit, S. de Groot, T. W. J. Janssen. Acquisition of data: K. J. A. Legemate, A. de Koning, C. A. J. Smit. Analysis and interpretation of data: C. A. J. Smit, S. de Groot, T. W. J. Janssen.

Drafting of manuscript: C. A. J. Smit, K. J. A. Legemate,

A. de Koning.

Critical revision of manuscript for important intellectual content:

C. A. J. Smit, J. M. Stolwijk-Swuste, S. de Groot, T. W. J. Janssen.

Statistical analysis: C. A. J. Smit, S. de Groot.

Study supervision: S. de Groot, T. W. J. Janssen.

Financial Disclosures: The authors have declared that no competing interests exist.

Funding/Support: This material was unfunded at the time of manuscript preparation.

Institutional Review: The local institutional medical ethical board of Reade Rehabilitation Research Centre and Slotervaart Hospital,

Amsterdam, the Netherlands, approved this study.

Participant Follow-Up: The authors do not plan to inform participants of the publication of this study. However, participants have been encouraged to check the study Web site for updated publications.

\section{REFERENCES}

1. Byrne DW, Salzberg CA. Major risk factors for pressure ulcers in the spinal cord disabled: A literature review. Spinal Cord. 1996;34(5):255-63. [PMID:8963971] http://dx.doi.org/10.1038/sc.1996.46

2. Liu LQ, Nicholson GP, Knight SL, Chelvarajah R, Gall A, Middleton FR, Ferguson-Pell MW, Craggs MD. Interface pressure and cutaneous hemoglobin and oxygenation changes under ischial tuberosities during sacral nerve root stimulation in spinal cord injury. J Rehabil Res Dev. 2006; 43(4):553-64. [PMID:17123193] http://dx.doi.org/10.1682/JRRD.2005.08.0135

3. Bogie KM, Wang X, Triolo RJ. Long-term prevention of pressure ulcers in high-risk patients: A single case study of the use of gluteal neuromuscular electric stimulation. Arch Phys Med Rehabil. 2006;87(4):585-91. [PMID:16571402] http://dx.doi.org/10.1016/j.apmr.2005.11.020

4. Ferguson AC, Keating JF, Delargy MA, Andrews BJ. Reduction of seating pressure using FES in patients with spinal cord injury. A preliminary report. Paraplegia. 1992; 30(7):474-78. [PMID:1508561]

http://dx.doi.org/10.1038/sc.1992.101

5. Rischbieth H, Jelbart M, Marshall R. Neuromuscular electrical stimulation keeps a tetraplegic subject in his chair: A case study. Spinal Cord. 1998;36(6):443-45.

[PMID:9648203]

http://dx.doi.org/10.1038/sj.sc.3100621

6. Levine SP, Kett RL, Cederna PS, Brooks SV. Electric muscle stimulation for pressure sore prevention: Tissue shape variation. Arch Phys Med Rehabil. 1990;71(3):210-15. [PMID:2180391]

7. Levine SP, Kett RL, Cederna PS, Bowers LD, Brooks SV. Electrical muscle stimulation for pressure variation at the seating interface. J Rehabil Res Dev. 1989;26(4):1-8. [PMID:2600864]

8. Levine SP, Kett RL. Tissue shape and deformation as a characterization of the seating interface. Proceedings of the Annual International Conference of the IEEE Engineering in Medicine and Biology Society; 1989 Nov 9-12; Seattle, WA. New York (NY): IEEE; 1989. p. 851-52.

9. Gerrits HL, Hopman MT, Sargeant AJ, Jones DA, De Haan A. Effects of training on contractile properties of paralyzed quadriceps muscle. Muscle Nerve. 2002;25(4):559-67. [PMID:11932974] http://dx.doi.org/10.1002/mus.10071

10. Reenalda J, Van Geffen P, Nederhand M, Jannink M, IJzerman M, Rietman H. Analysis of healthy sitting behavior: Interface pressure distribution and subcutaneous tissue oxygenation. J Rehabil Res Dev. 2009;46(5):577-86. [PMID:19882492] http://dx.doi.org/10.1682/JRRD.2008.12.0164

11. van Drongelen S, de Groot S, Veeger HE, Angenot EL, Dallmeijer AJ, Post MW, van der Woude LH. Upper extremity musculoskeletal pain during and after rehabilitation in wheelchair-using persons with a spinal cord injury. Spinal Cord. 2006;44(3):152-59. [PMID:16151450] http://dx.doi.org/10.1038/sj.sc.3101826

12. van Londen A, Herwegh M, van der Zee CH, Daffertshofer A, Smit CA, Niezen A, Janssen TW. The effect of surface electric stimulation of the gluteal muscles on the interface pressure in seated people with spinal cord injury. Arch Phys Med Rehabil. 2008;89(9):1724-32.

[PMID:18760157] http://dx.doi.org/10.1016/j.apmr.2008.02.028

13. Thomas CK, Griffin L, Godfrey S, Ribot-Ciscar E, Butler JE. Fatigue of paralyzed and control thenar muscles 
induced by variable or constant frequency stimulation. J Neurophysiol. 2003;89(4):2055-64. [PMID:12611940] http://dx.doi.org/10.1152/jn.01002.2002

14. Dreibati B, Lavet C, Pinti A, Poumarat G. Influence of electrical stimulation frequency on skeletal muscle force and fatigue. Ann Phys Rehabil Med. 2010;53(4):266-77. [PMID:20430713] http://dx.doi.org/10.1016/j.rehab.2010.03.004

15. Janssen T, Smit C, Hopman M. Prevention and treatment of pressure ulcers using electrical stimulation. In: Bader DL, Bouten CV, Colin D, Oomens CW, editors. Pressure ulcer research: Current and future perspectives. New York (NY): Springer; 2005. p. 89-108.

16. Smit CA, Haverkamp GL, de Groot S, Stolwijk-Swuste JM, Janssen TW. Effects of electrical stimulation-induced gluteal versus gluteal and hamstring muscles activation on sitting pressure distribution in persons with a spinal cord injury. Spinal Cord. 2012;50(8):590-94. [PMID:22350033] http://dx.doi.org/10.1038/sc.2012.6

17. Levine SP, Kett RL, Wilson BA, Cederna PS, Gross MD, Juni JE. Ischial blood flow of seated individuals during electrical muscle stimulation. Proceedings of the 10th Annual Conference on Rehabilitation Technology; 1987 Jun 19-23; San Jose, CA. Washington (DC): RESNA; 1987. p. 642-44.

18. Reenalda J, van Geffen P, Snoek G, Jannink M, Ijzerman M, Rietman H. Effects of dynamic sitting interventions on tissue oxygenation in individuals with spinal cord disorders. Spinal Cord. 2010;48(4):336-41. [PMID:19859080] http://dx.doi.org/10.1038/sc.2009.138
19. Janssen TW, Hopman MT. Blood flow response to electrically induced twitch and tetanic lower-limb muscle contractions. Arch Phys Med Rehabil. 2003;84(7):982-87.

[PMID:12881821]

http://dx.doi.org/10.1016/S0003-9993(03)00037-6

20. Bogie KM, Triolo RJ. Effects of regular use of neuromuscular electrical stimulation on tissue health. J Rehabil Res Dev. 2003;40(6):469-75. [PMID:15077659] http://dx.doi.org/10.1682/JRRD.2003.11.0469

21. Rosenthal MJ, Felton RM, Hileman DL, Lee M, Friedman M, Navach JH. A wheelchair cushion designed to redistribute sites of sitting pressure. Arch Phys Med Rehabil. 1996;77(3):278-82. [PMID:8600872] http://dx.doi.org/10.1016/S0003-9993(96)90112-4

Submitted for publication September 22, 2012. Accepted in revised form January 23, 2013.

This article and any supplementary material should be cited as follows:

Smit CA, Legemate KJ, de Koning A, de Groot S, Stolwijk-Swuste JM, Janssen TW. Prolonged electrical stimulation-induced gluteal and hamstring activation and sitting pressure in spinal cord injury: Effect of duty cycle. J Rehabil Res Dev. 2013;50(7):1035-46. http://dx.doi.org/10.1682/JRRD.2012.07.0134

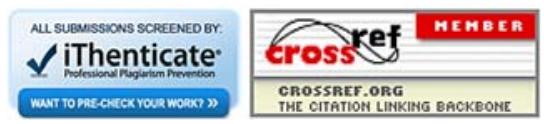


